\title{
On the link between rotation, chromospheric activity and Li abundance in subgiant stars
}

\author{
J. D. do Nascimento Jr. ${ }^{1}$, B. L. Canto Martins ${ }^{1}$, C. H. F. Melo ${ }^{2,1}$, \\ G. Porto de Mello ${ }^{3}$, and J. R. De Medeiros ${ }^{1}$
}

1 Departamento de Física, Universidade Federal do Rio Grande do Norte, 59072-970 Natal, RN., Brazil

2 European Southern Observatory, Casilla 19001, Santiago 19, Chile

3 Observatório do Valongo, Ladeira do Pedro Antonio, 43, 20080-090, Rio de Janeiro, RJ., Brazil

Received 14 October 2002 / Accepted 15 April 2003

\begin{abstract}
The connection rotation-CaII emission flux-lithium abundance is analyzed for a sample of bona fide subgiant stars, with evolutionary status determined from HIPPARCOS trigonometric parallax measurements and from the Toulouse-Geneva code. The distribution of rotation and CaII emission flux as a function of effective temperature shows a discontinuity located around the same spectral type, F8IV. Blueward of this spectral type, subgiants have a large spread of values of rotation and CaII flux, whereas stars redward of F8IV show essentially low rotation and low CaII flux. The strength of these declines depends on stellar mass. The abundance of lithium also shows a sudden decrease. For subgiants with mass lower than about $1.2 M_{\odot}$ the decrease is located later than that in rotation and CaII flux, whereas for masses higher than $1.2 M_{\odot}$ the decrease in lithium abundance is located around the spectral type F8IV. The discrepancy between the location of the discontinuities of rotation and CaII emission flux and $\log n(\mathrm{Li})$ for stars with masses lower than $1.2 M_{\odot}$ seems to reflect the sensitivity of these phenomena to the mass of the convective envelope. The drop in rotation, which results mostly from a magnetic braking, requires an increase in the mass of the convective envelope less than that required for the decrease in $\log n(\mathrm{Li})$. The location of the discontinuity in $\log n(\mathrm{Li})$ for stars with masses higher than $1.2 M_{\odot}$, in the same region of the discontinuities in rotation and CaII emission flux, may also be explained by the behavior of the deepening of the convective envelope. The more massive the star is, the earlier is the increase of the convective envelope. In contrast to the relationship between rotation and CaII flux, which is fairly linear, the relationship between lithium abundance and rotation shows no clear tendency toward linear behavior. Similarly, no clear linear trend is observed in the relationship between lithium abundance and CaII flux. In spite of these facts, subgiants with high lithium content also have high rotation and high CaII emission flux.
\end{abstract}

Key words. stars: activity - stars: abundances - stars: rotation - stars: interiors - stars: late-type

\section{Introduction}

The study of the influence of stellar rotation on chromospheric activity and on the mixing of light elements in evolved stars has undergone some important advances during the past decade. Several authors have reported a rotation-activity relation for evolved stars based on the linear behavior of the chromospheric flux with stellar rotation (e.g. Rutten 1987; Rutten \& Pylyser 1988; Simon \& Drake 1989; Strassmeier et al. 1994; Gunn et al. 1998; Pasquini et al. 2000). For a given spectral type, however, a large spread in the rotation-activity relation is observed, which suggests that rotation might not be the only relevant parameter controlling stellar activity. Indeed, results from Pasquini \& Brocato (1992) and Pasquini et al. (2000) have shown that chromospheric activity depends on stellar effective temperature and mass.

Send offprint requests to: J. D. do Nascimento Jr, e-mail: do.nascimento@dfte.ufrn.br
A possible connection between rotation and abundance of lithium in evolved stars has also been reported in the literature (e.g. De Medeiros et al. 1997; do Nascimento et al. 2000; De Medeiros et al. 2000). Subgiant and giant stars with enhanced lithium abundance show also enhanced rotation, in spite of a large spread in the abundances of lithium among the slow rotators. In addition, do Nascimento et al. (2000) have pointed to a discontinuity in the distribution of $\mathrm{Li}$ abundances as a function of effective temperature later than the discontinuity in rotation (e.g. De Medeiros \& Mayor 1990). Concerning the link between chromospheric activity and light element abundances, Duncan (1981) and Pasquini et al. (1994) have found a clear tendency of solar G-type stars with enhanced CaII surface flux $F(\mathrm{CaII})$ to have a higher lithium content. This is consistent with the predictions of standard evolutionary models, according to which, activity and abundance of light elements should depend on stellar surface temperature, metallicity and age. In spite of these important studies showing evidence of a 
connection in between abundance of lithium and rotation and in between chromospheric activity and rotation, in practice, for evolved stars, the mechanisms controlling such connections and their dependence on different stellar parameters like metallicity, mass and age are not yet well established. In this paper, we analyze in parallel the behavior of the chromospheric activity, stellar rotation and lithium abundance along the subgiant branch. In the present approach, the stars are placed in the HR diagram to determine more clearly the location of the discontinuities for these three stellar parameters based on a sample of bona fide subgiants.

\section{Working sample}

For this study we have selected a large sample of 121 single stars classified as subgiants in the literature, along the spectral region $\mathrm{F}, \mathrm{G}$ and $\mathrm{K}$, with rotational velocity, flux of $\mathrm{CaII}$ and $\log n(\mathrm{Li})$ now available. The rotational velocities $v \sin i$ were taken from De Medeiros \& Mayor (1999). By using the CORAVEL spectrometer (Baranne et al. 1979) these authors have determined the projected rotational velocity $v \sin i$ for a large sample of subgiant and giant stars with a precision of about $1 \mathrm{~km} \mathrm{~s}^{-1}$ for stars with $v \sin i$ lower than about $30 \mathrm{~km} \mathrm{~s}^{-1}$. For higher rotators, the estimations indicate an uncertainty of about $10 \%$. The $F$ (CaII) was determined from the $\mathrm{CaII} \mathrm{H}$ and K line-core emission index $S_{1}$ and $S_{2}$ listed by Rutten (1987), using the procedure of conversion from the emission index S1 to flux at the stellar surface $F$ (CaII) given by Rutten (1984). The values of $\log n(\mathrm{Li})$ were taken from Lèbre et al. (1999) and Randich et al. (1999). Readers are referred to these works for discussion on the observational procedure, data reduction and error analysis. Stellar luminosities were determined as follows. First, the apparent visual magnitudes $m_{\mathrm{v}}$ and trigonometric parallaxes, both taken from HIPPARCOS catalogue (ESA 1997), were combined to yield the absolute visual magnitude $M_{\mathrm{v}}$. Bolometric correction $B C$, computed from Flower (1996) calibration, was applied giving the bolometric magnitude which was finally converted into stellar luminosity. The effective temperature was computed using Flower (1996) $(B-V)$ versus $T_{\text {eff }}$ calibration. The rotational velocity $v \sin i$, stellar surface flux $F(\mathrm{CaII})$, abundance of lithium $\log n(\mathrm{Li})$ and stellar parameters of the entire sample are presented in Table 1.

\section{Results}

\subsection{The discontinuity in rotation, Call emission flux and $L i$ abundance}

As a first step, the stellar luminosity and the effective temperature listed in Table 1 were used to construct the HR diagram to better locate the evolutionary stage of the stars in the sample. In fact, such a procedure seems important because in preceding studies on the link between rotation and chromospheric activity in subgiant stars, only the spectral type was used as a criterion for identifying the stars. Evolutionary tracks were computed from the Toulouse-Geneva code for stellar masses between 1 and $4 M_{\odot}$, for metallicity consistent with solar-type subgiant stars (see do Nascimento et al. 2000 for a more detailed

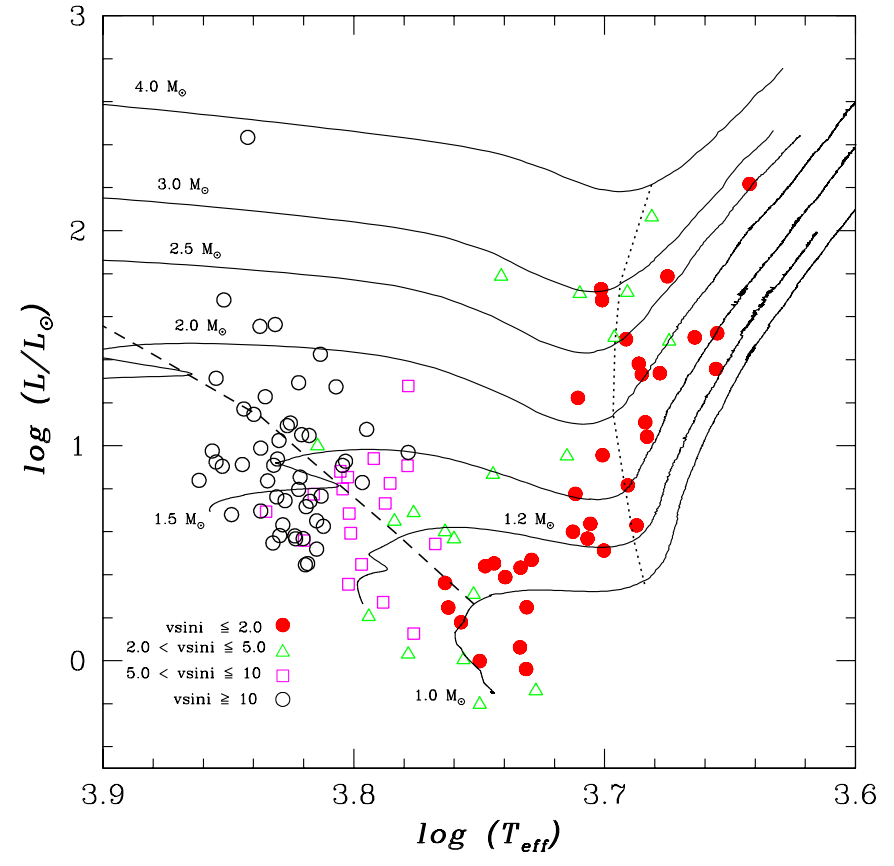

Fig. 1. Distribution of subgiant stars in the HR diagram, with the rotational behavior as a function of luminosity and effective temperature. Luminosities have been derived from the HIPPARCOS parallaxes. Evolutionary tracks at $[\mathrm{Fe} / \mathrm{H}]=0$ are shown for stellar masses between 1 and $4 M_{\odot}$. The dashed line indicates the beginning of the subgiant branch and the dotted line represents the beginning on the red giant branch.

description). Here, in particular, we use the evolutionary tracks computed with solar metallicity because most of the stars in the present sample have $[\mathrm{Fe} / \mathrm{H}] \sim 0$. The HR diagram with the evolutionary tracks is displayed in Figs. 1, 2 and 3, which in addition show the behavior of the rotational velocity $v \sin i$, surface flux CaII and $\log n(\mathrm{Li})$ abundance respectively. In these diagrams the dashed line indicates the evolutionary region where the subgiant branch starts, corresponding to hydrogen exhaustion in stellar central regions, whereas the dotted line represents the beginning of the ascent of the red giant branch. One observes, clearly, that most of the stars in the present sample are effectively subgiants. Nevertheless a small number of stars located in particular on the cool side of the diagrams are rather stars evolving along the red giant branch. In this context, for the purpose of the present analysis, these deviating stars will not be considered as subgiants, in spite of the spectral types assigned in the literature.

Figure 1 shows the well established rotational discontinuity around the spectral type F8IV (e.g. De Medeiros \& Mayor $1990)$, corresponding to $(B-V) \approx 0.55\left(\log T_{\text {eff }} \sim 3.78\right)$. As shown by these authors, single subgiants blueward of this spectral type show a wide range of rotational velocities from a few $\mathrm{km} \mathrm{s}^{-1}$ to about one hundred times the solar rotation, whereas subgiants redward of F8IV are essentially slow rotators, except for the synchronized binary systems. Figure 1 shows clearly that single subgiants redward of the discontinuity with high $v \sin i$ are unusual. The root cause for such a discontinuity seems to be a strong magnetic braking associated with 
Table 1. The stars of the present workimg sample with their physical parameters.

\begin{tabular}{|c|c|c|c|c|c|c|}
\hline $\mathrm{HD}$ & ST & $\log (L / L o)$ & $T_{\text {eff }}$ & $v \sin i$ & $F(\mathrm{CaII})$ & $\log n(\mathrm{Li})$ \\
\hline 400 & F8IV & 0.45 & 6265 & 5.6 & 6.635 & $2.30^{a}$ \\
\hline 645 & K0IV & 1.33 & 4844 & 1.8 & 5.551 & $0.50^{a}$ \\
\hline 905 & FOIV & 0.68 & 7059 & 31.6 & 7.137 & \\
\hline 3229 & F5IV & 1.00 & 6524 & 5.0 & 6.932 & $1.30^{a}$ \\
\hline 4744 & G8IV & 1.49 & 4724 & 3.4 & 5.326 & \\
\hline 4813 & F7IV-V & 0.21 & 6223 & 3.9 & 6.658 & $2.80^{a}$ \\
\hline 5268 & G5IV & 1.68 & 5024 & 1.9 & 5.646 & $0.40^{a}$ \\
\hline 5286 & K1IV & 1.04 & 4821 & 1.6 & 5.573 & \\
\hline 6301 & F7IV-V & 0.65 & 6528 & 20.3 & 6.829 & $1.00^{a}$ \\
\hline 6680 & F5IV & 0.63 & 6735 & 36.4 & 7.086 & \\
\hline 8799 & F5IV & 0.85 & 6628 & 65.9 & 6.934 & \\
\hline 9562 & G2IV & 0.57 & 5755 & 4.2 & 6.327 & $2.40^{a}$ \\
\hline 11151 & F5IV & 0.80 & 6637 & 34.0 & 6.834 & \\
\hline 12235 & G2IV & 0.54 & 5855 & 5.2 & 6.423 & $1.30^{a}$ \\
\hline 13421 & G0IV & 0.91 & 6006 & 9.9 & 6.415 & $1.30^{a}$ \\
\hline 13871 & F6IV-V & 0.77 & 6546 & 9.1 & 6.763 & \\
\hline 16141 & G5IV & 0.31 & 5653 & 2.3 & 6.269 & \\
\hline 18262 & F7IV & 0.80 & 6375 & 9.9 & 6.621 & $2.10^{b}$ \\
\hline 18404 & F5IV & 0.57 & 6656 & 24.7 & 6.902 & \\
\hline 20618 & G8IV & 1.22 & 5137 & 1.0 & 5.984 & \\
\hline 23249 & K0IV & 0.51 & 5015 & 1.0 & 5.770 & $0.90^{b}$ \\
\hline 25621 & F6IV & 0.83 & 6261 & 15.3 & 6.758 & $3.01^{b}$ \\
\hline 26913 & G5IV & -0.20 & 5621 & 3.9 & 6.646 & $2.20^{a}$ \\
\hline 26923 & G0IV & 0.03 & 6002 & 4.3 & 6.712 & $2.80^{a}$ \\
\hline 29859 & F7IV-V & 0.83 & 6103 & 9.0 & 6.457 & \\
\hline 30912 & F2IV & 1.56 & 6877 & $155^{f}$ & 6.914 & \\
\hline 33021 & G1IV & 0.36 & 5803 & 2.0 & 6.357 & $2.00^{a}$ \\
\hline 34180 & FOIV & 0.74 & 6721 & $80^{f}$ & 7.015 & \\
\hline 34411 & G2IV-V & 0.25 & 5785 & 1.9 & 6.360 & $2.00^{a}$ \\
\hline 37788 & FOIV & 0.92 & 7160 & 31.2 & 7.196 & \\
\hline 39881 & G5IV & 0.18 & 5718 & 1.4 & 6.329 & \\
\hline 43386 & F5IV-V & 0.45 & 6582 & 18.8 & 6.927 & $2.30^{b}$ \\
\hline 53329 & G8IV & 1.73 & 5028 & 1.3 & 5.702 & \\
\hline 57749 & F3IV & 2.43 & 6955 & $40^{f}$ & 6.759 & \\
\hline 60532 & F6IV & 0.94 & 6195 & 8.1 & 6.590 & $1.60^{a}$ \\
\hline 64685 & F2IV & 0.70 & 6873 & 67.2 & 7.087 & \\
\hline 66011 & G0IV & 0.97 & 6002 & 13.6 & 6.489 & $1.20^{a}$ \\
\hline 71952 & K0IV & 1.11 & 4828 & 1.0 & 5.520 & \\
\hline 73017 & G8IV & 1.50 & 4915 & 1.2 & 5.618 & \\
\hline 73593 & G0IV & 1.38 & 4857 & 1.0 & 5.561 & \\
\hline 76291 & K1IV & 1.50 & 4614 & 1.2 & 5.282 & \\
\hline 78154 & F7IV-V & 0.59 & 6328 & 5.8 & 6.600 & $1.10^{a}$ \\
\hline 81937 & FOIV & 1.15 & 6916 & $145^{f}$ & 7.084 & \\
\hline 82074 & G6IV & 0.95 & 5188 & 2.1 & 5.951 & $0.30^{a}$ \\
\hline 82328 & F6IV & 0.88 & 6388 & 8.3 & 6.751 & $3.30^{a}$ \\
\hline 82734 & K0IV & 2.06 & 4800 & 3.8 & 5.413 & $1.10^{a}$ \\
\hline 84117 & F9IV & 0.27 & 6142 & 5.6 & 6.627 & $2.50^{b}$ \\
\hline 89449 & F6IV & 0.63 & 6488 & 17.3 & 6.763 & $1.30^{a}$ \\
\hline 92588 & K1IV & 0.57 & 5091 & 1.0 & 5.863 & $1.00^{a}$ \\
\hline 94386 & K3IV & 1.36 & 4525 & 1.0 & 5.133 & $0.20^{a}$ \\
\hline 99028 & F2IV & 1.05 & 6619 & 16.0 & 7.015 & $3.25^{b}$ \\
\hline 99329 & F3IV & 0.91 & 6989 & $130^{f}$ & 7.186 & \\
\hline 99491 & K0IV & -0.14 & 5338 & 2.6 & 6.206 & $1.40^{a}$ \\
\hline
\end{tabular}

Sources: ${ }^{a}$ - Lèbre et al. (1999); ${ }^{b}$ - De Medeiros et al. (1997); ${ }^{c}-$ Randich et al. (1999); ${ }^{f}$ - Uesugi \& Fukuda (1982). 
Table 1. continued.

\begin{tabular}{|c|c|c|c|c|c|c|}
\hline HD & ST & $\log (L / L o)$ & $T_{\mathrm{eff}}$ & $v \sin i$ & $F(\mathrm{CaII})$ & $\log n(\mathrm{Li})$ \\
\hline 104055 & K2IV & 2.22 & 4388 & 2.0 & 5.003 & $0.20^{a}$ \\
\hline 104304 & KOIV & -0.04 & 5387 & 2.0 & 6.127 & $0.90^{a}$ \\
\hline 105678 & F6IV & 1.08 & 6236 & 29.6 & 6.766 & $1.60^{a}$ \\
\hline 107326 & FOIV & 0.98 & 7185 & $120^{f}$ & 7.191 & \\
\hline 110834 & F6IV & 1.27 & 6414 & $145^{f}$ & 6.880 & \\
\hline 117361 & FOIV & 1.09 & 6707 & $85^{f}$ & 6.973 & \\
\hline 119992 & F7IV-V & 0.36 & 6341 & 8.3 & 6.624 & $2.70^{a}$ \\
\hline 121146 & K2IV & 1.52 & 4520 & 1.0 & 5.116 & \\
\hline 123255 & F2IV & 1.17 & 6980 & $140^{f}$ & 7.119 & \\
\hline 124570 & F6IV & 0.73 & 6130 & 5.6 & 6.494 & $2.80^{a}$ \\
\hline 125111 & F2IV & 0.69 & 6839 & 9.3 & 7.075 & \\
\hline 125184 & G5IV & 0.39 & 5491 & 1.3 & 6.229 & $0.80^{a}$ \\
\hline 125451 & F5IV & 0.55 & 6796 & 46.0 & 7.048 & $1.80^{a}$ \\
\hline 125538 & G9IV & 1.79 & 4731 & 1.0 & 5.363 & \\
\hline 126943 & F1IV & 0.99 & 6873 & $80^{f}$ & 7.078 & \\
\hline 127243 & G3IV & 1.71 & 5128 & 3.6 & 5.802 & $0.60^{a}$ \\
\hline 127739 & F2IV & 0.94 & 6768 & 68.0 & 6.991 & \\
\hline 127821 & F4IV & 0.45 & 6596 & 45.5 & 6.954 & \\
\hline 130945 & F7IVw & 0.93 & 6358 & 18.7 & 6.689 & $2.30^{b}$ \\
\hline 133484 & F6IV & 0.77 & 6502 & 21.2 & 6.786 & $2.70^{a}$ \\
\hline 136064 & F9IV & 0.65 & 6079 & 5.0 & 6.511 & $2.00^{a}$ \\
\hline 143584 & FOIV & 0.84 & 7273 & $70^{f}$ & 7.271 & \\
\hline 145148 & K0IV & 0.63 & 4867 & 1.0 & 5.612 & $0.00^{c}$ \\
\hline 150012 & F5IV & 1.05 & 6573 & 35.5 & 6.968 & $2.50^{a}$ \\
\hline 154160 & G5IV & 0.47 & 5360 & 1.2 & 5.856 & $1.60^{a}$ \\
\hline 154417 & F8.5IV-V & 0.13 & 5972 & 5.9 & 6.723 & \\
\hline 156697 & F0-2IV-Vn & 1.56 & 6782 & $160^{f}$ & 6.931 & \\
\hline 156846 & G3IV & 0.69 & 5972 & 4.9 & 6.468 & $0.80^{a}$ \\
\hline 157347 & G5IV & 0.00 & 5621 & 1.1 & 6.360 & $0.70^{c}$ \\
\hline 157853 & F8IV & 1.79 & 5511 & 3.2 & 6.488 & $2.20^{a}$ \\
\hline 158170 & F5IV & 1.28 & 6002 & 8.0 & 6.587 & $1.20^{a}$ \\
\hline 161797 & G5IV & 0.43 & 5414 & 1.7 & 6.109 & $1.10^{a}$ \\
\hline 162003 & F5IV-V & 0.74 & 6569 & 12.9 & 6.795 & $2.60^{a}$ \\
\hline 162076 & G5IV & 1.50 & 4967 & 3.2 & 5.959 & $1.10^{a}$ \\
\hline 162917 & F4IV-V & 0.57 & 6610 & $50^{f}$ & 6.891 & \\
\hline 164259 & F2IV & 0.76 & 6772 & $80^{f}$ & 6.997 & \\
\hline 165438 & K1IV & 0.82 & 4907 & 1.0 & 5.647 & $0.12^{c}$ \\
\hline 173949 & G7IV & 1.71 & 4909 & 2.6 & 5.614 & \\
\hline 176095 & F5IV & 0.91 & 6375 & 13.2 & 6.795 & $2.90^{a}$ \\
\hline 182572 & G8IV & 0.25 & 5384 & 1.7 & 6.135 & \\
\hline 182640 & FOIV & 0.90 & 7119 & 68.4 & 7.154 & \\
\hline 184663 & F6IV & 0.58 & 6660 & 69.0 & 6.992 & $1.90^{b}$ \\
\hline 185124 & F3IV & 0.72 & 6592 & $85^{f}$ & 6.943 & \\
\hline 188512 & G8IV & 0.78 & 5148 & 1.2 & 5.905 & $0.10^{c}$ \\
\hline 190360 & G6IV+M6V & 0.06 & 5417 & 1.7 & 6.197 & \\
\hline 190771 & G5IV & 0.01 & 5705 & 2.7 & 6.685 & $2.30^{a}$ \\
\hline 191026 & K0IV & 0.60 & 5160 & 1.3 & 6.295 & \\
\hline 191570 & F5IV & 0.58 & 6754 & 33.6 & 7.008 & $2.60^{a}$ \\
\hline 192344 & G4IV & 0.45 & 5547 & 1.4 & 6.234 & \\
\hline 195564 & G2.5IV & 0.44 & 5593 & 1.9 & 6.185 & $1.97^{c}$ \\
\hline 196755 & $\mathrm{G} 5 \mathrm{IV}+\mathrm{K} 2 \mathrm{IV}$ & 0.87 & 5553 & 3.3 & 6.218 & $1.10^{a}$ \\
\hline 197373 & F6IV & 0.52 & 6528 & 30.9 & 6.816 & $1.00^{a}$ \\
\hline 197964 & K1IV & 1.34 & 4764 & 1.0 & 5.466 & \\
\hline
\end{tabular}

Sources: ${ }^{a}$ - Lèbre et al. (1999); ${ }^{b}$ - De Medeiros et al. (1997); ${ }^{c}$ - Randich et al. (1999); ${ }^{f}$ - Uesugi \& Fukuda (1982). 
Table 1. continued.

\begin{tabular}{clccccc}
\hline \hline HD & ST & $\log (L / L \mathrm{o})$ & $T_{\text {eff }}$ & $v \sin i$ & $F(\mathrm{CaII})$ & $\log n(\mathrm{Li})$ \\
\hline 198149 & K0IV & 0.96 & 5022 & 1.4 & 5.737 & \\
201507 & F5IV & 1.23 & 6844 & 16.4 & 7.051 & \\
201636 & F3IV & 0.91 & 6791 & 67.9 & 7.025 & \\
202444 & F1IV & 1.02 & 6758 & 26.1 & 7.039 & \\
202582 & G2IV+G2IV & 0.60 & 5803 & 3.1 & 6.479 & $2.20^{a}$ \\
205852 & F1IV & 1.68 & 7109 & $180^{f}$ & 7.127 & \\
207978 & F6IV-Vvw & 0.56 & 6605 & 7.2 & 6.770 & $1.00^{a}$ \\
208703 & F5IV & 0.84 & 6829 & 15.4 & 7.078 & \\
210210 & F1IV & 1.31 & 7160 & $80^{f}$ & 7.089 & \\
212487 & F5IV & 0.85 & 6345 & 8.8 & 6.582 & $2.20^{b}$ \\
216385 & F7IV & 0.68 & 6336 & 5.9 & 6.610 & $1.20^{a}$ \\
218101 & G8IV & 0.64 & 5078 & 1.1 & 6.096 & \\
219291 & F6IVw & 1.43 & 6506 & 53.1 & 6.944 & \\
223421 & F2IV & 1.11 & 6688 & 66.6 & 7.001 & \\
224617 & F4IV & 1.29 & 6637 & 49.9 & 6.913 & $3.20^{b}$ \\
\hline
\end{tabular}

Sources: ${ }^{a}$ - Lèbre et al. (1999); ${ }^{b}$ - De Medeiros et al. (1997); ${ }^{c}-$ Randich et al. (1999); ${ }^{f}-$ Uesugi \& Fukuda (1982).

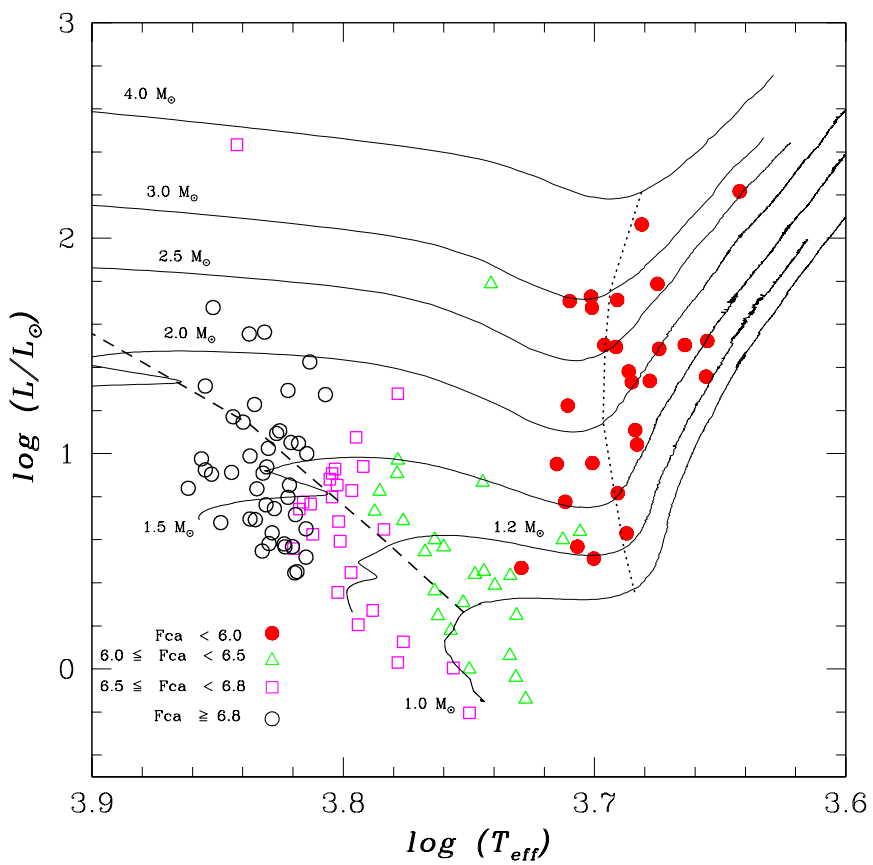

Fig. 2. Distribution of subgiant stars in the HR diagram, with the behavior of the $F(\mathrm{CaII})$ surface flux as a function of luminosity and effective temperature. Luminosities have been derived from the HIPPARCOS parallaxes. Evolutionary tracks are defined as in Fig. 1.

the rapid increase of the moment of inertia, due to evolutionary expansion, once the star evolves along the late $\mathrm{F}$ spectral region (e.g. Gray \& Nagar 1985; De Medeiros \& Mayor 1990).

Figure 2 shows clear evidence of a discontinuity in the surface flux $F(\mathrm{CaII})$ paralleling the one observed in rotational velocity. In fact, such a sudden decrease in CaII flux of subgiants also parallels that in CIV emission flux found by Simon \& Drake (1989). Stars with typical subgiant masses showing the highest CaII flux are located blueward of this discontinuity. Such a drop in the surface chromospheric flux is interpreted by Simon \& Drake (1989) as the result of the drop in rotation

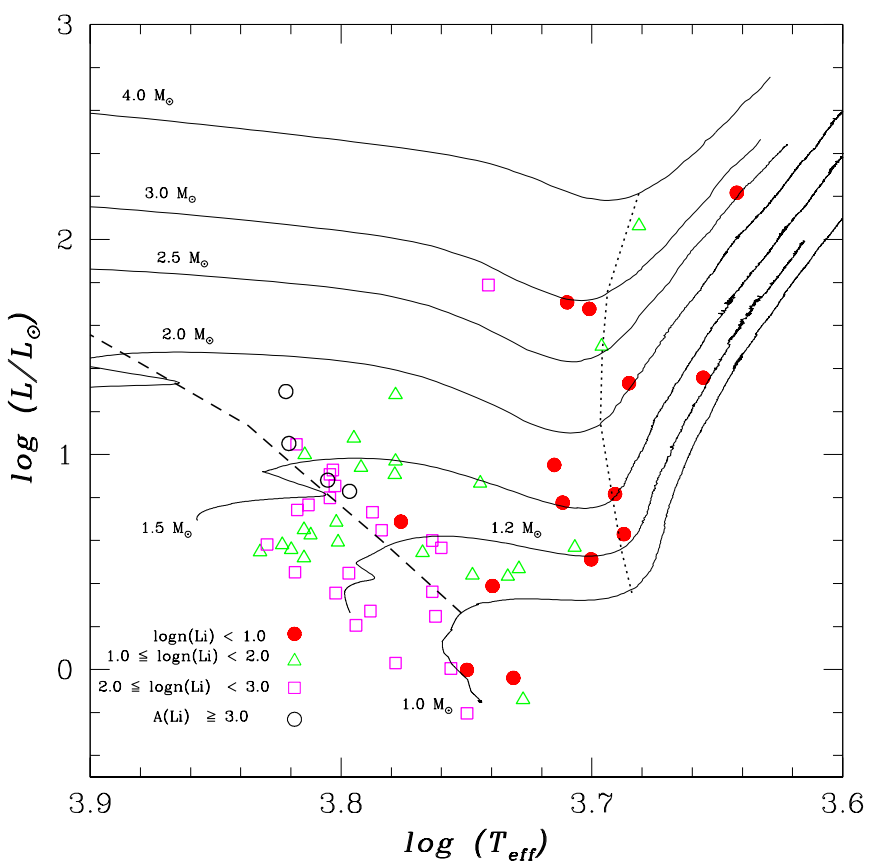

Fig. 3. Distribution of subgiant stars in the HR diagram, with the behavior of Li abundance as a function of luminosity and effective temperature. Luminosities have been derived from the HIPPARCOS parallaxes. Evolutionary tracks are defined as in Fig. 1.

near the spectral type G0IV. According to these authors, there is a development of a dynamo in late $\mathrm{F}$ stars, which induces a strong magnetic braking in a preexisting wind that acts on the outermost layers of the stellar surface. As a consequence the stellar surface will spin down.

Figure 3 shows the behavior of the lithium abundance, with a sudden decrease in $\log n(\mathrm{Li})$ for subgiant stars with mass lower than about $1.2 M_{\odot}$, located a somewhat later than the discontinuity in rotation and in surface $F$ (CaII). Evidence for this decrease in $\log n(\mathrm{Li})$ was first pointed out by do Nascimento et al. (2000). According to these authors, such a drop in 


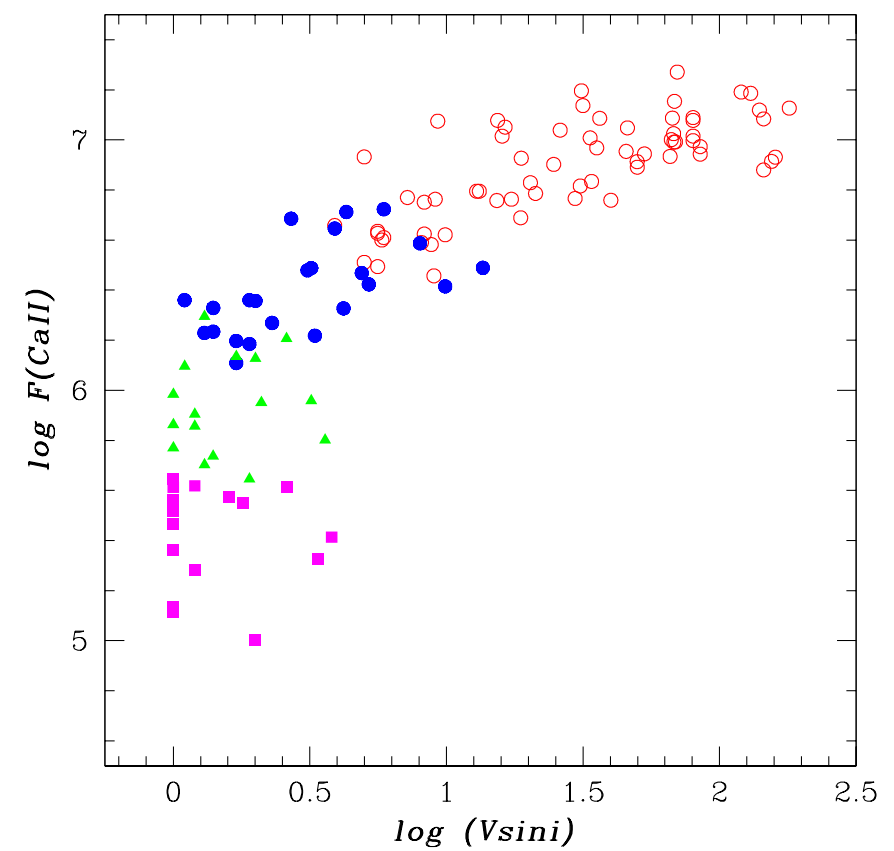

Fig. 4. $\log F(\mathrm{CaII})$ versus $\log (v \sin i)$ for the program stars. Open circles denote stars with $(B-V) \leq 0.55$, filled circles those with $0.55<(B-V) \leq 0.75$, triangles stars with $0.75<(B-V) \leq 0.95$ and squares stars with $(B-V)>0.95$.

$\log n(\mathrm{Li})$ abundances of subgiants seems to result from the rapid increase of the convective envelope at the late $\mathrm{F}$ evolutionary stage. Due to the convective mixing process, $\mathrm{Li}-$ rich surface material is diluted towards the stellar interior. For higher masses, the drop in $\log n(\mathrm{Li})$ shows a tendency to parallel the discontinuities in $v \sin i$ and $F(\mathrm{CaII})$, near F8IV, corresponding to $(B-V) \approx 0.55\left(\log T_{\text {eff }} \sim 3.78\right)$.

An additional trend is present in Figs. 1 and 2, which show that the fastest rotators and those subgiants with the highest CaII emission flux, namely the stars blueward of F8IV, are mostly stars with mass higher than about $1.2 M_{\odot}$. Subgiants with mass lower than about $1.2 M_{\odot}$ show moderate to low rotation as well as moderate to low surface $F(\mathrm{CaII})$. In the region blueward of F8IV, the abundances of lithium show a more complex behavior for stars with masses between 1.2 and $1.5 \mathrm{M}_{\odot}$. Figure 3 shows a number of stars in this mass interval with low to moderate $\log n(\mathrm{Li})$. Such a fact appears to reflect the socalled dip region observed by Boesgaard \& Tripicco (1986).

\subsection{The relation Rotation $-F($ Call $)-\log n(\mathrm{Li})$}

As a second step of this study we have analyzed the direct relationship between rotation, $F(\mathrm{CaII})$ and $\log n(\mathrm{Li})$ for the stars of the sample. Figure 4 shows the surface $F(\mathrm{CaII})$ versus the rotational velocity $v \sin i$, where stars are separated by intervals of $(B-V)$. Stars earlier than the rotational discontinuity, typically those with $(B-V) \leq 0.55$, are represented by open circles, solid circles stand for stars with $0.55<(B-V) \leq 0.75$, triangles stand for stars with $0.75<(B-V) \leq 0.95$ and squares represent stars with $(B-V)>0.95$. The well established correlation between rotation and chromospheric emission flux (e.g.

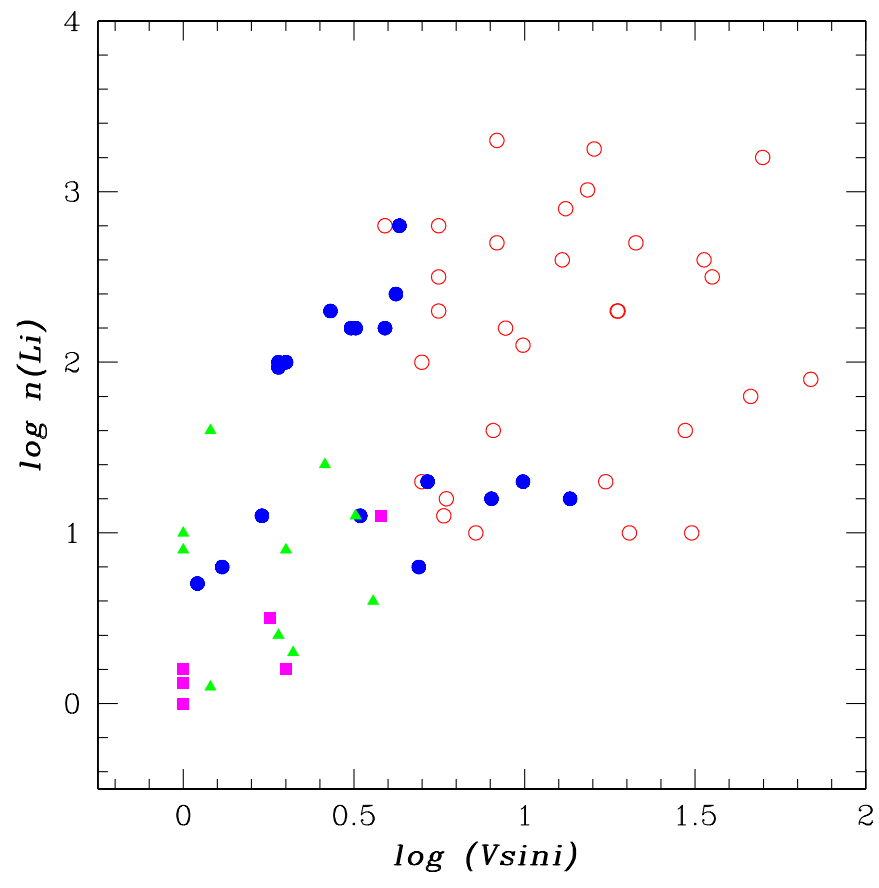

Fig. 5. $\log n(\mathrm{Li})$ versus $\log (v \sin i)$ for the program stars. Symbols are defined as in Fig. 4.

Simon \& Drake 1989), here represented by the surface $F(\mathrm{CaII})$, is clearly confirmed for the present sample of bona fide subgiants.

Figure 5 presents the behavior of $\log n(\mathrm{Li})$ as a function of the rotational velocity $v \sin i$, confirming the trend of a fair connection in between abundance of Li and $v \sin i$ in subgiant stars already observed by other authors (e.g. De Medeiros et al. 1997).

Finally, Fig. 6 shows the surface $F(\mathrm{CaII})$ as a function of $\log n(\mathrm{Li})$. In spite of more a limited number of stars than in Figs. 4 and 5, we observe a trend for a connection between $F(\mathrm{CaII})$ and $\log n(\mathrm{Li})$ following rather the behavior observed in the $v \sin i$ versus $\log n(\mathrm{Li})$ relation.

\subsection{The connection F(Call) emission flux-Rossby number}

A close examination of the rotation versus $F(\mathrm{CaII})$ relation presented in Fig. 4 shows that the amount by which it deviates from a linear correlation depends on the $(B-V)$ color interval. A similar color dependence was observed by Noyes et al. (1984), who removed such an effect by introducing the dimensionless Rossby number $R_{0}=P_{\text {rot }} / \tau_{\text {conv }}$, as a mesure of the rotational velocity. This dependence was also noted by Simon \& Drake (1989) for subgiant stars, by analysing the relation $F(\mathrm{CIV})$ versus rotation. These results confirm that rotation is not the only parameter expected to influence stellar chromospheric activity; another is the stellar mass, or equivalently, the position of the star in the HR diagram, which dictates the properties of the stellar convective zone. The deepening of the convective zone, or its convective turnover time is, in particular, expected to play a relevant role in the dynamo generation. The Rossby number, in fact, determines the extent to which rotation can induce both helicity and differential rotation 


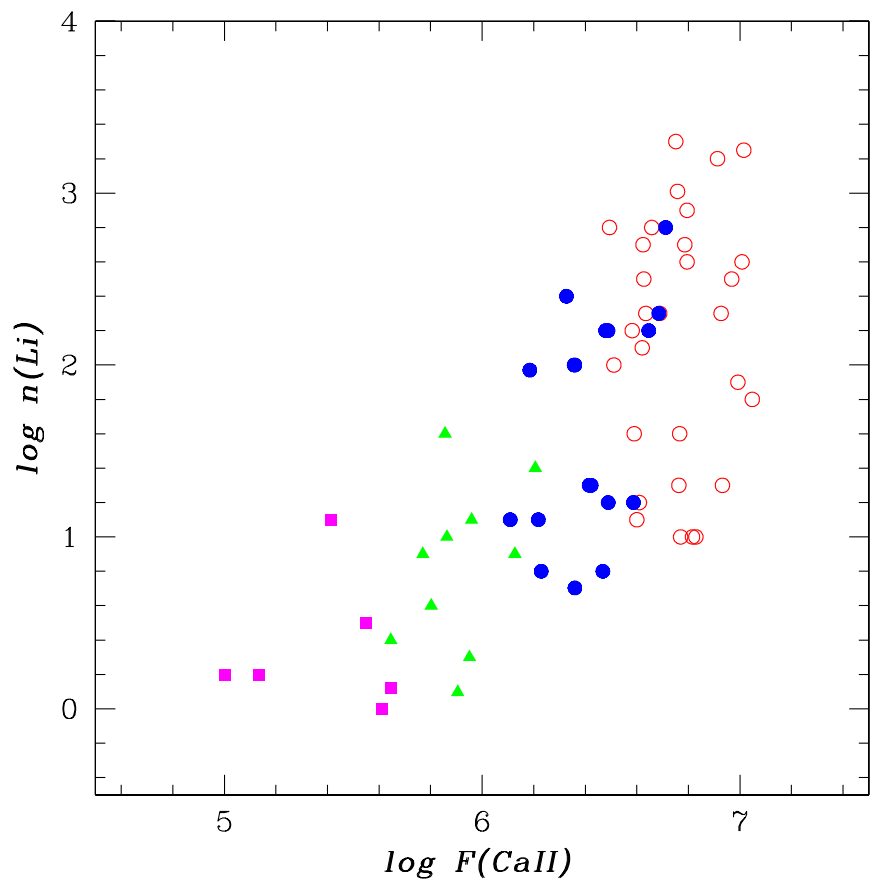

Fig. 6. $\log n(\mathrm{Li})$ versus $\log F(\mathrm{CaII})$ for the program stars. Symbols are defined as in Fig. 4.

required for dynamo activity in the convective zone. To analyse the connection $F$ (CaII) emission flux-Rossby number, we have computed $R_{0}$ for all the stars of the present sample. The convective turnover time $\tau_{\text {conv }}$ was estimated from the iterated function in $(B-V)$ given by Noyes et al. (1984), whereas the rotation period was estimated indirectly from the $v \sin i$ given in Table 1. A statistical correction of $\pi / 4$ was taken in consideration, to compensate for $\sin i$ effects. The stellar radii were estimated following the standard expression as a function of effective temperature and luminosity. Figure 7 presents the behavior of $F(\mathrm{CaII})$ as a function of the Rossby number $R_{0}$, with two clear different features. For stars with $(B-V)>0.55$ the correlation of chromospheric activity, given by $F(\mathrm{CaII})$, with $R_{0}$ is significantly better than with rotational velocity, whereas stars with $(B-V) \leq 0.55$ show $F$ (CaII) rather uniformly high and independent of the $R_{0}$. A similar result was found by Simon $\&$ Drake (1989), by analysing the $F(\mathrm{CIV})$ versus $R_{0}$ relation.

\subsection{The behavior of $\log n(\mathrm{Li})$ as a function of the deepening of the convective envelope}

The level of dilution of lithium depends strongly on the level of convection. In this context it sounds interesting to analyse the behavior of lithium abundance as a function of the deepening of the convective zone for the present sample of stars. For this purpose we have estimated the mass of each star $M_{*}$ from the HR diagram presented in Sect. 3.1 and then estimated the mass of the convective zone $M_{\mathrm{CZ}}$ from an iterated function $M_{\mathrm{CZ}}\left(M_{*}, T_{\text {eff }}\right)$ constructed on the basis of the study by do Nascimento et al. (2000) on the deepening (in mass) of the convective envelope of evolved stars. These authors present the behavior of $M_{\mathrm{CZ}}$ as a function of $T_{\mathrm{eff}}$ for stars with masses

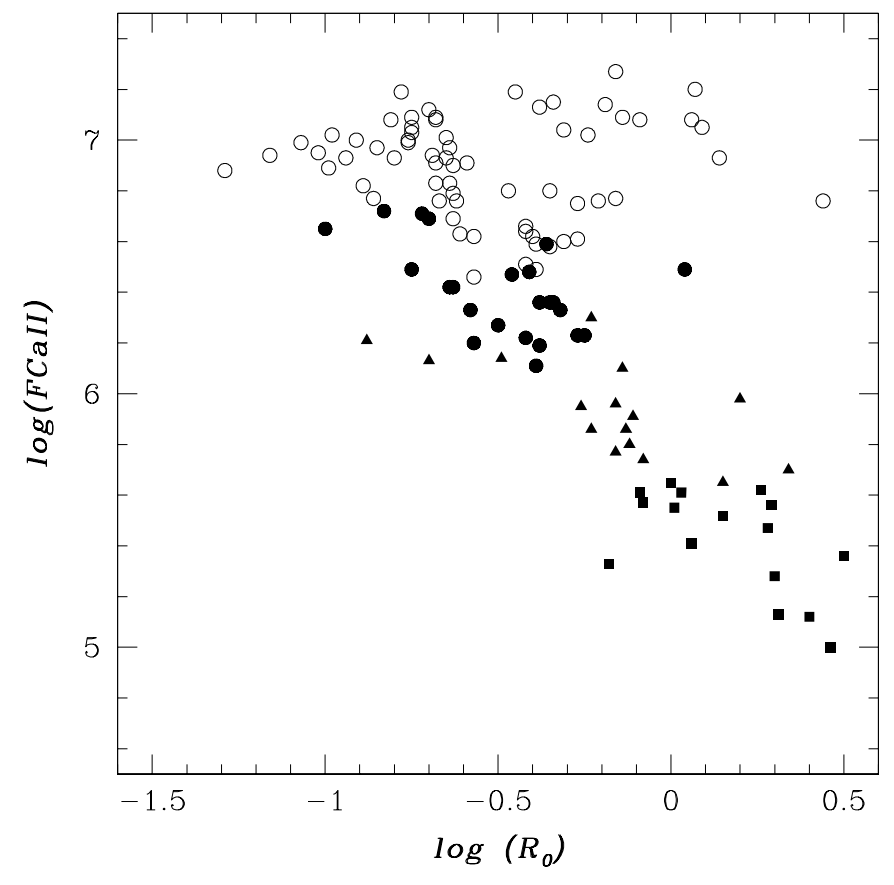

Fig. 7. The $F(\mathrm{CaII})$ versus the Rossby number $R_{0}$. The symbols are defined as in Fig. 4.

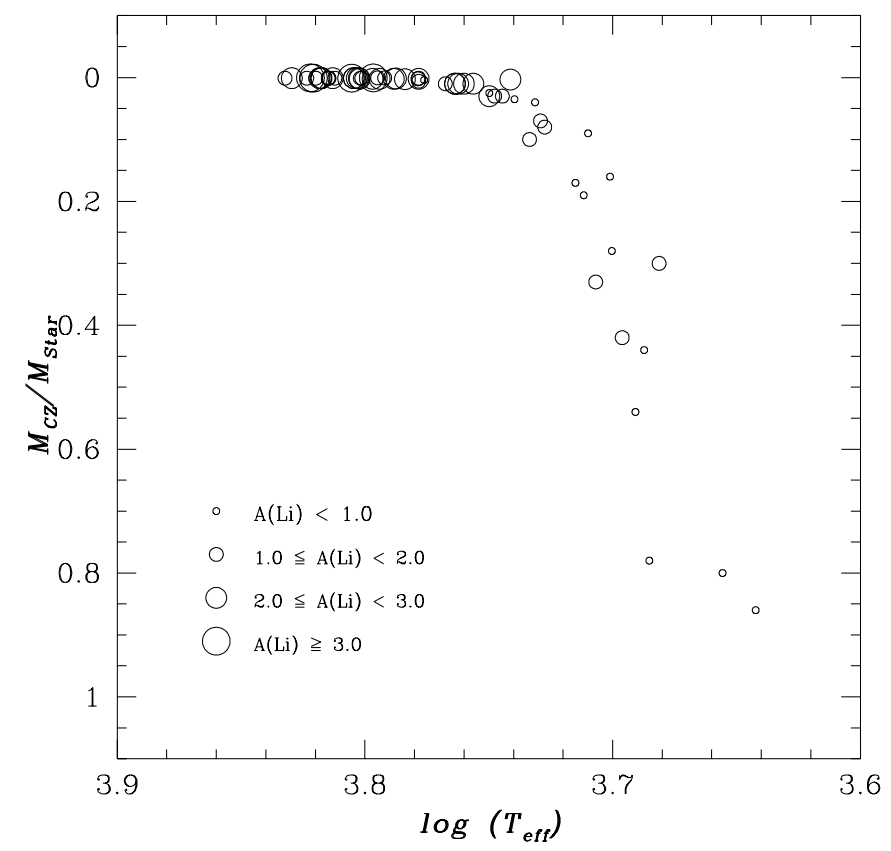

Fig. 8. The deepening (in mass) of the convective envelope as a function of the effective temperature for the stars in the present sample. The symbol size is proportional to the $\mathrm{Li}$ abundances quoted in.

between 1.0 and $4.0 M_{\odot}$. Figure 8 shows the behavior of $\log n(\mathrm{Li})$ in the $M_{\mathrm{CZ}} / M_{*}$ versus $T_{\text {eff }}$ diagram. It is clear that most of the stars with high lithium content present an undeveloped convective envelope, whereas stars with low $\log n(\mathrm{Li})$ have a developed convective envelope. 


\section{Discussion}

At this point we can inquire about the root cause of the apparent discrepancy in the location of the discontinuities in $v \sin i$ and $F(\mathrm{CaII})$ and that for $\log n(\mathrm{Li})$. In fact, should one expect, from the evolutionary point of view, that the discontinuity in $\log n(\mathrm{Li})$ follows the one in $v \sin i$ as well as that in $F(\mathrm{CaII})$ ? First of all, let us recall that in the specific case of late-type evolved stars, chromospheric activity reflects the presence of magnetic fields which are relevant for the heating of the chromosphere as well as for mass and angular momentum losses. The intensity and spatial distribution of magnetic fields are very probably determined by a dynamo process, whose mode of operation and efficiency depends on the interplay between stellar rotation and subphotospheric convective motions. In this context one should expect a direct link between the discontinuities in $v \sin i$ and $F(\mathrm{CaII})$, with a drop at the same spectral region, if chromospheric activity is directly controlled by rotation. As shown by Fig. 7, this is true, in particular, for stars located redward of the spectral region of the discontinuity.

The question now turns to the apparent discrepancy in the location of the discontinuity in $\log n(\mathrm{Li})$ in relation to the location of the discontinuities in $v \sin i$ and $F$ (CaII) for subgiant stars with masses lower than about $1.2 M_{\odot}$. This discrepancy can be understood as a result of the sensitivity of these phenomena to the mass of the convective envelope. In the case of the rotational discontinuity, a small increase in the mass of the convective envelope is enough to turn the dynamo on. This same dynamo will be responsible to the magnetic braking causing a drop in the rotation rate and the consequent shutdown of the dynamo itself. Later, the convective envelope will continue to deep reaching a region previously devoid of $\mathrm{Li}$. At this point, the Li brought from the surface layers is diluted and its abundance drops. This fact explains clearly the discrepancy between the location of the discontinuity in $\log n(\mathrm{Li})$ in relation to the one for $v \sin i$ and $F$ (CaII), as observed from Figs. 1 to 3 . The fact that a magnetic braking might operate with very small changes in the mass of the convective envelope is further reinforced by the location of the discontinuity in the $F(\mathrm{CaII})$ flux at the late F spectral region. Previous studies (e.g. do Nascimento et al. 2000) show that the development of the convective envelope towards the stellar interior starts at this spectral region, reaching a maximum within the middle to late $G$ spectral region. In short, the drop in $v \sin i$ and $F(\mathrm{CaII})$ is earlier than that in $\log n(\mathrm{Li})$ because, in contrast to the former, this latter requires a large increase in the mass of the convective envelope. Figure 8 shows that Li dilution increases abruptly with the deepening of the convective envelope. In fact, the observed discontinuity in $\log n(\mathrm{Li})$ seems to be controlled directly by the increasing of the deepening of the convective envelope.

The observed trend for a same location, of the discontinuities in $v \sin i$ and $\log n(\mathrm{Li})$ for stars with masses larger than about $1.2 M_{\odot}$ may also be explained by following the behavior of the deepening of the convective envelope. As shown by do Nascimento et al. (2000, see their Fig. 4), the changes in the mass of the convective envelope at a given effective temperature in the range from $\log T_{\text {eff }} \sim 3.75$ to $\log T_{\text {eff }} \sim 3.68$, are more important for stars with masses in the increasing sequence of masses from $1.0 M_{\odot}$ to $2.5 M_{\odot}$. The more massive the star is, in this range of masses, the earlier is the increasing of the convective envelope. In this context, a sudden decrease in $\log n(\mathrm{Li})$ of stars with masses larger than about 1.2 $M_{\odot}$, paralleling the rotational discontinuity, should be expected.

The relationship between $v \sin i$ and surface $F(\mathrm{CaII})$, as presented in Fig. 4, confirms the results found by other authors for subgiant stars (e.g. Strassmeier et al. 1994) and for other luminosity classes (Strassmeier et al. 1994; Pasquini et al. 2000). In addition, one observes a trend of increasing scattering in the $v \sin i$ versus $F(\mathrm{CaII}$ ) relation, confirming previous claims that rotation might not be the only relevant parameter controlling chromospheric activity. In this context, Pasquini et al. (2000) have found for giant stars a clear dependence of $F(\mathrm{CaII})$ flux with a high power of stellar effective temperature, whereas Strassmeier et al. (1994) have found that the CaII flux from the cooler evolved stars depends more strongly upon rotation than the CaII flux from the hotter evolved stars. The behavior of $F(\mathrm{CaII})$ as a function of the Rossby number $R_{0}$, presented in Fig. 7, shows two clear trends: For stars with $(B-V)$ larger than about 0.55 the $F(\mathrm{CaII})$ tends towards a linear correlation with $R_{0}$; stars with $(B-V)$ lower than about 0.55 show $F(\mathrm{CaII})$ rather uniformly high and independent of $R_{0}$, pointing for a component of chromospheric activity independent of rotation. Different authors (e.g. Wolff et al. 1986) suggest that the chromospheres of early F stars may be heated by the shock dissipation of sound waves, rather than by the dynamo process that control the chromospheric activity in $\mathrm{G}$ - and $\mathrm{K}$-type stars.

The dependence of lithium abundance upon rotation observed in Fig. 5 exists in the sense that the fastest rotators also have the highest lithium content. Nevertheless, there is no clear linear relation between these two parameters. Figure 5 also shows a large spread in the Li content at a given $v \sin i$ value, covering at least 2 mag in $\log n(\mathrm{Li})$. Such a spread shows a clear tendency to increase with rotation and effective temperature. For $v \sin i$ lower than about $10 \mathrm{~km} \mathrm{~s}^{-1}$, in particular, the $\log n(\mathrm{Li})$ values range from about 0.0 to about 3.0. Such a spread was also observed by De Medeiros et al. (1997) \& do Nascimento et al. (2000). Finally, the behavior of $\log n(\mathrm{Li})$ as a function of CaII emission flux presented in Fig. 6 seems to follow roughly the same trend observed for the relation $v \sin i$ versus $\log n(\mathrm{Li})$. Subgiants with high lithium content also show high $F(\mathrm{CaII})$, but there is no clear linear relation between these two parameters.

\section{Summary and conclusions}

In the search for a better understanding of the influence of stellar rotation on chromospheric activity and lithium dilution, we have analyzed the relationship rotation-CaII emission flux$\mathrm{Li}$ abundance along the subgiant branch, on the basis of a sample of bona fide subgiants, reclassified from HIPPARCOS data. The evolutionary status of all the stars was determined from trigonometric parallax taken from this data base and evolutionary tracks computed from the Geneva-Toulouse code. The distributions of the rotational velocity and of the CaII emission flux show similar behavior. For both parameters we observe a sudden decrease around the spectral type F8IV, 
confirming previous studies. Nevertheless, the extent of these discontinuities depends on the stellar mass. Stars with masses around $1.5 M_{\odot}$ show a more important decrease in rotation and CaII emission flux, than stars with masses lower than about $1.2 M_{\odot}$. Clearly, stars blueward of F8IV, with masses higher than $1.2 M_{\odot}$, rotate faster and are more active than those with masses lower than about $1.2 M_{\odot}$. The distribution of Li abundance versus effective temperature, in spite of a sudden decrease in the late-F region shows a trend for a more complex behavior. First, stars with masses lower than about $1.2 M_{\odot}$ show a discontinuity in $\log n(\mathrm{Li})$ somewhat later than the discontinuities in rotation and CaII emission flux, whereas stars with higher masses present a decline in $\log n(\mathrm{Li})$ rather around the spectral type F8IV. In addition, a group of stars blueward of F8IV with masses between 1.2 and $1.5 M_{\odot}$ shows moderate to low $\log n(\mathrm{Li})$, which seems to reflect the effects of the socalled Boesgaard-Tipico dip region. The discrepancy in the location of the discontinuities of rotation-CaII emission flux and $\log n(\mathrm{Li})$ for stars with masses lower than $1.2 M_{\odot}$, seems to be the result of the sensitivity of these phenomena to the mass of the convective envelope. The drop in rotation, resulting mostly from a magnetic braking, requires an increase in the mass of the convective envelope less than that required for the sudden decrease in $\log n(\mathrm{Li})$, this later resulting from the dilution due to the rapid increase of the convective envelope. The location of the discontinuity in $\log n(\mathrm{Li})$ for stars with masses higher than $1.2 M_{\odot}$, in the same region of the discontinuities in rotation and CaII emission flux, may also be explained by following the behavior of the deepening of the convective envelope. The more massive the star is, the earlier is the increase of the convective envelope. The present work confirms that the dilution of Li depends strongly on the deepening of the convective envelope.

The relationship between rotation and CaII emission flux confirms previous results found by other authors. CaII emission flux shows a correlation with rotation. Nevertheless, the large spread in the CaII flux $-v \sin i$ relation reinforces previous suggestions that rotation might not be the only relevant parameter controlling stellar chromospheric activity. In fact, the relation $F$ (CaII) versus Rossby number confirms that chromospheric activity of subgiant stars with $(B-V)$ larger than about 0.55 depends rather linearly on rotation, whereas for stars with $(B-V)$ lower than about 0.55 activity is rather independent of rotation. The relationship between $\log n(\mathrm{Li})$ and rotation shows a behavior less clear than that between CaII flux and rotation. Of course the present study confirms a dependence of lithium abundance upon rotation, in the sense that stars with the high rotation have also high lithium content. In spite of this fact, there is no clear linear relationship between these two parameters, with a spread more important than that observed in the $F(\mathrm{CaII})-v \sin i$ relation. The behavior of the relationship between lithium abundance and CaII emission flux seems to follow that observed for $\log n(\mathrm{Li})-v \sin i$. Stars with the high activity also show high lithium content. In both cases there is a remarkable increase in scattering in the $\log n(\mathrm{Li})-v \sin i$ and $\log n(\mathrm{Li})-\mathrm{CaII}$ flux relations with increasing $v \sin i$ and CaII flux, respectively. Such a fact appears to indicate that the influence of rotation on stellar activity is greater than on lithium dilution. Finally, the present study point to a pressing need for new measurements of chromospheric emission flux and lithium abundance for an homogeneous and larger sample of bona fide subgiant stars, with a larger range of metallicities, than that analyzed here. With these additional data it will be possible to analyze the influence of rotation upon activity and lithium dilution on a more solid basis, taking into account the stellar age and metallicity.

Acknowledgements. This work has been supported by continuous grants from the CNPq Brazilian Agency. J.D.N.Jr. acknowledges the CNPq grant PROFIX 540461/01-6. Special thanks to the referee, Dr. R. Cayrel for very useful comments, which greatly improved the quality of this paper.

\section{References}

Baranne, A., Mayor, M., \& Poncet, J. L. 1979, Vistas Astron., 23, 279 Boesgaard, A. M., \& Trippico, M. J. 1986, ApJ, 302, L49

De Medeiros, J. R., \& Mayor, M. 1990, Cool stars, stellar systems, and the sun; Proceedings of the 6th Cambridge Workshop, Seattle, Astronomical Society of the Pacific, 6, 404

De Medeiros, J. R., do Nascimento, J. D. Jr., \& Mayor, M. 1997, A\&A, 317,701

De Medeiros, J. R., \& Mayor, M. 1999, A\&AS, 139, 433

De Medeiros, J. R., do Nascimento, J. D. Jr., Sankarankutty, S., Costa, J. M., \& Maia, M. R. G. 2000, A\&A, 363, 239

do Nascimento, J. D. Jr., Charbonnel, C., Lèbre, A., de Laverny, P., \& De Medeiros, J. R. 2000, A\&A, 357, 931

Duncan, D. K. 1981, ApJ, 248, 651

ESA 1997, The Hipparcos and Tycho Catalogues, ESA SP-1200

Flower, P. J. 1996, ApJ, 469, 355

Gray, D. F., \& Nagar, P. 1985, ApJ, 298, 756

Gunn, A. G., Mitrou, C. K., \& Doyle, J. G. 1998, MNRAS, 296, 150

Lèbre, A., de Laverny, P., De Medeiros, J. R., Charbonnel, C., \& da Silva, L. 1999, A\&A, 345, 936

Noyes, R. W., Hartmann, L. W., Baliunas, S. L., Duncan, D. K., \& Vaughan, A. H. 1984, ApJ, 279, 763

Pasquini, L., \& Brocato, E. 1992, A\&A, 266, 340

Pasquini, L., Liu, Q., \& Pallavicini, R. 1994, A\&A, 287, 191

Pasquini, L., De Medeiros, J. R., \& Girardi, L. 2000, A\&A, 361, 1011

Randich, S., Gratton, R., Pallavicini, R., Pasquini, L., \& Carretta, E. 1999, A\&A, 348, 487

Rutten, R. G. M. 1984, A\&A, 130, 353

Rutten, R. G. M. 1987, A\&A, 177, 131

Rutten, R. G. M., \& Pylyser, E. 1988, A\&A, 191, 227

Simon, T., \& Drake, S., A. 1989, ApJ, 346, 303

Strassmeier, K. G., Handler, G., Paunzen, E., \& Rauth, M. 1994, A\&A, 281, 855

Uesugi, A., \& Fukuda, I. 1982, Catalogue of stellar rotational velocities (revised)

Wollf, S. C., Boesgaard, A. M., \& Simon, T. 1986, ApJ, 310, 360 